\title{
Correction to: Understanding the Origins of Problem Geomagnetic Storms Associated with "Stealth" Coronal Mass Ejections
}

Nariaki V. Nitta ${ }^{1}$ - Tamitha Mulligan² ${ }^{2}$ Emilia K.J. Kilpua ${ }^{3} \cdot$ Benjamin J. Lynch $^{4}$. Marilena Mierla ${ }^{5,6}$. Jennifer O'Kane ${ }^{7}$ Paolo Pagano ${ }^{8,9,10}$. Erika Palmerio ${ }^{3,4,11}$. Jens Pomoell ${ }^{3}$. Ian G. Richardson ${ }^{12,13}$ • Luciano Rodriguez ${ }^{5}$. Alexis P. Rouillard ${ }^{14}$. Suvadip Sinha ${ }^{15}$. Nandita Srivastava ${ }^{16,15}$. Dana-Camelia Talpeanu ${ }^{17,5}$. Stephanie L. Yardley ${ }^{8,7}$. Andrei N. Zhukov ${ }^{5,18}$

Published online: 17 November 2021

(c) The Author(s) 2021

Correction to: Space Sci. Rev. (2021) 217: 82

https://doi.org/10.1007/s11214-021-00857-0

Unfortunately, a mistake was introduced in the name of author Ian G. Richardson. The original article has been corrected.

Publisher's Note Springer Nature remains neutral with regard to jurisdictional claims in published maps and institutional affiliations. 


\section{Authors and Affiliations}

Nariaki V. Nitta ${ }^{1}$ D Tamitha Mulligan² - Emilia K.J. Kilpua ${ }^{3}$ - Benjamin J. Lynch ${ }^{4}$. Marilena Mierla ${ }^{5,6}$. Jennifer O'Kane ${ }^{7}$ Paolo Pagano ${ }^{8,9,10}$. Erika Palmerio ${ }^{3,4,11}$. Jens Pomoell ${ }^{3}$ Ian G. Richardson ${ }^{12,13}$. Luciano Rodriguez ${ }^{5}$. Alexis P. Rouillard ${ }^{14}$. Suvadip Sinha ${ }^{15}$. Nandita Srivastava ${ }^{16,15}$. Dana-Camelia Talpeanu ${ }^{17,5}$. Stephanie L. Yardley ${ }^{8,7}$. Andrei N. Zhukov ${ }^{5,18}$

$\bigotimes$ N.V. Nitta

nitta@1msal.com

Lockheed Martin Solar and Astrophysics Laboratory, Palo Alto, CA 94304, USA

Space Sciences Department, The Aerospace Corporation, Los Angeles, CA 94305, USA

Department of Physics, University of Helsinki, FI-00014 Helsinki, Finland

Space Sciences Laboratory, University of California-Berkeley, Berkeley, CA 94720, USA

5 Solar-Terrestrial Centre of Excellence-SIDC, Royal Observatory of Belgium, 1180 Brussels, Belgium

6 Institute of Geodynamics of the Romanian Academy, 020032 Bucharest-37, Romania

7 Mullard Space Science Laboratory, University College London, Holmbury St. Mary, Dorking, Surrey RH5 6NT, UK

8 School of Mathematics and Statistics, University of St Andrews, North Haugh, St Andrews, Fife KY16 9SS, UK

9 Dipartimento di Fisica \& Chimica, Università di Palermo, I-90134 Palermo, Italy

10 INAF-Osservatorio Astronomico di Palermo, I-90134 Palermo, Italy

11 Cooperative Programs for the Advancement of Earth System Science, University Corporation for Atmospheric Research, Boulder, CO 80301, USA

Department of Astronomy, University of Maryland, College Park, MD 20742, USA

Centre of Excellence in Space Sciences India, Indian Institute of Science Education and Research Kolkata, Mohanpur 741246, India

16 Udaipur Solar Observatory, Physical Research Laboratory, Udaipur 313001, India

17 Centre for Mathematical Plasma Astrophysics (CmPA), KU Leuven, 3001 Leuven, Belgium

18 Skobeltsyn Institute of Nuclear Physics, Moscow State University, 119991 Moscow, Russia 